\title{
Structure-Property Model for Polyethylene-Derived Carbon Fiber
}

Michael J. Behr ${ }^{1 *}$, Brian G. Landes ${ }^{1}$, Bryan E. Barton ${ }^{1}$, Mark T. Bernius ${ }^{1}$, Gerry F. Billovits ${ }^{1}$, Eric J. Hukkanen ${ }^{1}$, Jasson T. Patton ${ }^{1}$, Weijun Wang ${ }^{1}$, Charlie Wood ${ }^{1}$; Denis T. Keane ${ }^{2}$, James E. Rix $^{2}$, Steven J. Weigand ${ }^{2}$

${ }^{1}$ Core Research and Development, The Dow Chemical Company, Midland, MI 48667

${ }^{2}$ DND-CAT Synchrotron Research Center, Northwestern University, APS/ANL 432-A004, 9700 S. Cass Avenue, Argonne, IL 60439

\section{Abstract}

This paper presents a structure-property model for carbon fiber derived from a polyethylene (PE) precursor that relates tensile modulus to the elastic properties and angular distribution of constituent graphitic layers, as measured using wide-angle x-ray diffraction of individual carbon fiber filaments. The observed relationship and interpretation of data using a uniform-stress model has revealed fundamental differences in the nature of the microstructure present in carbon fiber produced from polyethylene compared to carbon fiber produced from polyacrylonitrile (PAN) or pitch precursors. Specifically, it was found that the shear modulus, indicative of the shear between adjacent graphitic layers of the carbonized fiber is lower for polyethylene-derived carbon fiber than for PAN- or pitch-derived carbon fiber, suggesting that the covalent C-C $\mathrm{sp}^{3}$ crosslink density connecting adjacent graphitic layers in PE-derived carbon fiber is reduced. This structure that is less crosslinked is anticipated to be easier to orient during carbonization and high-temperature graphitization processes, yielding a highly oriented structure necessary for high tensile modulus.

\section{Introduction}

Carbon fiber exhibits a unique combination of extraordinary attractive material properties, including high specific tensile modulus and strength, high temperature creep resistance, chemical

* Corresponding author. Tel: 989 636-2583. E-mail: mjbehr@dow.com (Michael Behr). 
resistance, and low thermal expansion, that makes it an ideal material for structural reinforcement in light-weight composites for a wide range of applications, including aerospace, wind-turbine blades, infrastructure, automotive, pressure vessels, and sports equipment [1].

Currently, the majority of commercial carbon fiber is produced from polyacrylonitrile (PAN) [1]. Specifically, the PAN-based process employs an expensive monomer, challenging white fiber processes, and a relatively low mass yield ( $50 \%)$. Thus a significant opportunity exists to produce a new material-based, cost-advantaged, industrial-grade carbon fiber derived from a melt-spun polyethylene precursor, which is cost advantaged, due in part to its significantly higher carbon mass yield (60-80\%).

Transformation of polyethylene into carbon fiber has been demonstrated previously in the literature. For example, patent US4070446 (Sumitomo Chemical) described the transformation of high-density polyethylene to carbon fiber with a tensile modulus of $147 \mathrm{GPa}$, and a strength of 2.3 GPa [2]. More recently, Postema and Penning used linear-low-density polyethylene (LLDPE) to produce carbon fiber with a tensile modulus of $130 \mathrm{GPa}$, and a tensile strength of 2.2 GPa [3,4]. However, the most impressive previously published result is that of Zhang et al., who achieved a tensile modulus of $200 \mathrm{GPa}$ and strength of $2 \mathrm{GPa}$ from a gel spun, highly drawn, ultra-high molecular weight polyethylene [5]. Despite these successes, few microstructural details were reported, and thus many questions remain about the characteristics of the microstructure in the final carbonized fibers, and resulting structure-property relationship. Knowledge of this microstructure-property relationship is critical to guide precursor selection, and further stabilization and carbonization process development.

The extraordinary combined properties of carbon fiber are a direct consequence of its constituent highly-oriented graphitic microstructure. The fundamental building block of a carbon fiber, a two-dimensional sheet of hexagonally-arranged $\mathrm{sp}^{2}$-bonded carbon atoms, is similar to that of graphene, which exhibits incredible mechanical properties. Specifically, a single sheet of graphene exhibits $\sim 1010 \mathrm{GPa}$ tensile modulus, $107 \mathrm{GPa}$ tensile strength, and $20 \%$ strain at failure [6]. In a carbon fiber, these graphitic sheets are intertwined, well-connected, and preferentially aligned along the fiber axis, as shown pictorially in Figure 1. It is well-accepted 
that the degree of preferred orientation controls the resulting tensile modulus of the fiber. The degree of preferred orientation and the resulting tensile properties have been well-studied by numerous groups using wide-angle $\mathrm{x}$-ray diffraction of carbon fibers derived from PAN, mesophase pitch, and cellulose precursors [1, 7-13].

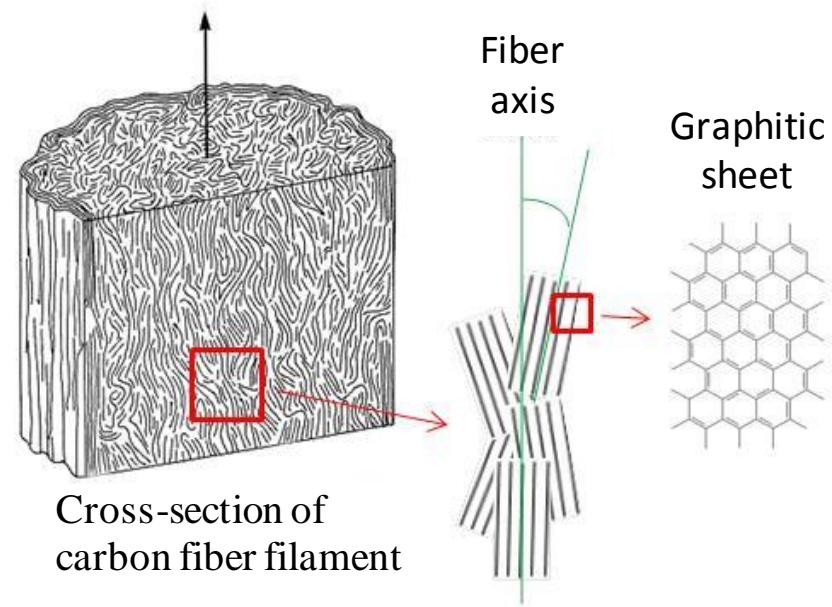

Figure 1. Pictorial representation of the aligned graphitic microstructure of a carbon fiber. Adapted from Morgan [1].

Models have been proposed in the literature, which relate the extensional tensile properties to the angular distribution of graphitic layers relative to the fiber axis to account for the high correlation observed between preferred orientation and tensile modulus [7, 11, 14, 15]. However, the specific relationships are found to depend on both the chemical and structural nature of the precursor fiber [7], as these factors influence the microstructure present in the final carbon fiber [1]. Specifically, these factors can influence the resulting modulus of shear between adjacent graphitic layers, and it is this shear modulus parameter that is critically important in determining the mechanical properties. Thus the relationship between tensile modulus and the graphitic layer orientation distribution for PAN fiber is different than that of pitch or cellulose due to differences in the modulus for shear between its constituent graphitic layers.

The purpose of this paper is to present a structure-property model that relates the tensile modulus to the elastic properties and angular distribution of constituent graphitic layers specifically for carbon fiber derived from polyethylene precursors. This model, first proposed by Ruland [11] and later established by Northolt et al. [7], not only provides predictive capability to determine 
the maximum attainable tensile properties for a given level of microstructure orientation, but more importantly also provides insights into the physical connectivity of the graphitic layers within the fiber through the shear modulus parameter. This information is critical to guide precursor fiber selection and processing, as well as optimization of the stabilization and carbonization processes.

\section{Theory}

The relationship between the tensile modulus and the orientation distribution of the constituent graphitic layers for the well-oriented microstructure of a carbon fiber can be interpreted using a uniform stress, or series model, as first described by Ruland [11]. In this model, the fiber is considered to be built up of a parallel array of fibrils that are well connected in the fiber direction, but weakly in the perpendicular direction; the applied tensile stress is thus distributed uniformly across these fibrils. This model, established by Northolt et al. [7] relates the tensile modulus to the orientation distribution of graphene layers relative to the fiber axis and the shear modulus for shear between adjacent layers through the equation

$$
\frac{1}{E}=\frac{1}{e_{1}}+\frac{\left\langle\cos ^{2} \varphi\right\rangle}{g}
$$

where $E$ is the tensile modulus, $e_{1}$ is the in-plane modulus of graphite (1060 GPa), $g$ is the shear modulus for shear between adjacent graphitic layers (4-5 GPa in graphite), and $\left\langle\cos ^{2} \varphi\right\rangle$ is the orientation distribution or average orientation of graphitic layers, defined by [12]

$$
\left\langle\cos ^{2} \varphi\right\rangle=\frac{\int_{0}^{\pi / 2} \rho(\varphi) \cos ^{2} \varphi \sin \varphi d \varphi}{\int_{0}^{\pi / 2} \rho(\varphi) \sin \varphi d \varphi}=\frac{\int_{0}^{\pi / 2} I(\varphi) \cos ^{2} \varphi \sin \varphi d \varphi}{\int_{0}^{\pi / 2} I(\varphi) \sin \varphi d \varphi}
$$

where $\rho(\varphi) \sin \varphi$ is the fraction of (002) diffraction peak (graphitic plane normals) with angles between $\varphi+d \varphi$, and $I(\varphi)$ is the intensity of the (002) diffraction peak at an angle $\varphi$. The angle $\varphi$ is defined as that between the graphitic layer plane normal (c-axis) and the fiber axis, as shown in Figure 2 below. The integral in the denominator in Eq. (2) is the total scattered intensity of the sample. This equation shows that fiber extension can be separated into two components: extension of the graphitic layers themselves, and shear between adjacent layers. Though more refined models have been proposed by Sauder et al [14], the Northolt model was found to be to be sufficient for the purpose of comparison of fibers from different precursors. 
The orientation parameter describes the average orientation of the graphitic layer plane normals (c-axis) relative to the fiber axis, as shown in Figure 2. However, it is the distribution of graphitic layer plane normals relative to the direction perpendicular to the fiber axis that is measured in a transmission $\mathrm{x}$-ray diffraction experiment, described in terms of the angle $\theta$ as shown in Figure 2 and 3. This is due to the absence of a unique, strongly-reflecting set of planes normal to the fiber axis direction [16]. Thus, the orientation parameter for alignment of graphitic layer plane normals relative to the fiber axis must be determined indirectly, as described in the experimental section. The relationship between the orientation distribution relative to the fiber axis and the perpendicular axis for a carbon fiber with cylindrical symmetry is given by [17]

$$
\left\langle\cos ^{2} \delta\right\rangle=1-2\left\langle\cos ^{2} \varphi\right\rangle
$$

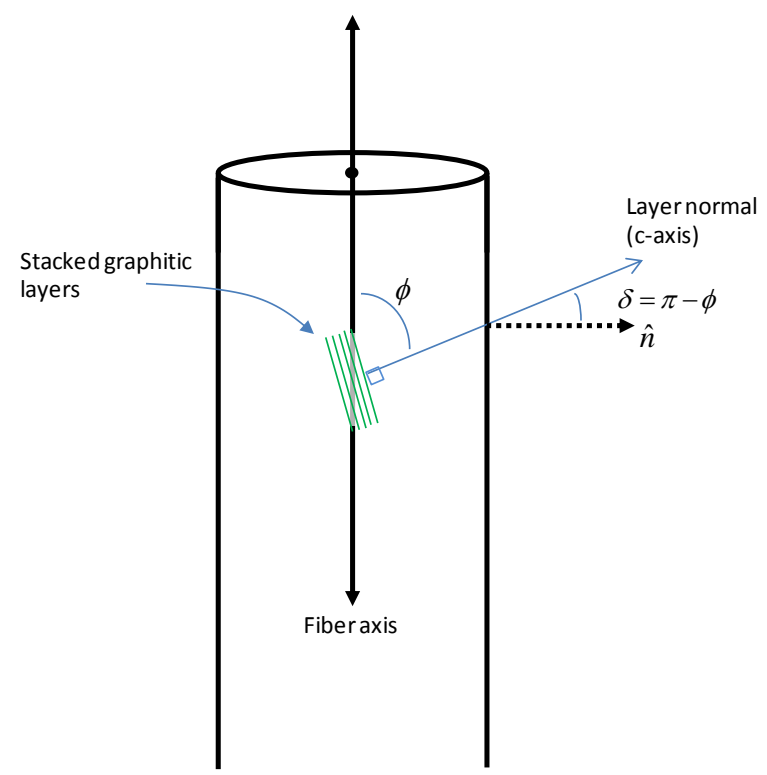

Figure 2. Schematic diagram of a stacked graphitic domain within a carbon fiber, with orientation angles defined. Adapted from Sauder et al. [18]. 

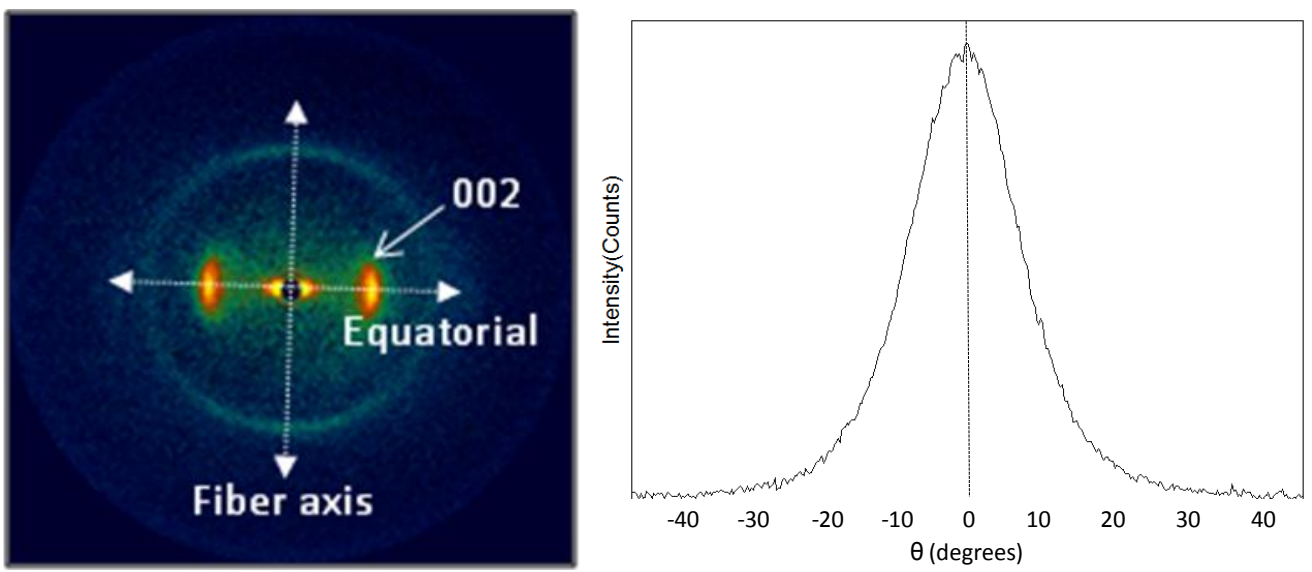

Figure 3. (Left) Wide-angle $x$-ray diffraction pattern obtained from a single carbon fiber filament. Orientation of graphitic layers along the fiber axis is evident from the anisotropic azimuthal intensity distribution of the 002 diffraction ring. (Right) Azimuthal intensity distribution of the right (002) diffraction ring relative to the equatorial direction.

\section{Materials and Experimental Procedures}

\subsection{Description of Materials}

The carbon fibers examined in this study were prepared using polyethylene resins sourced from The Dow Chemical Company [19]. Oriented precursor fibers with diameters less than 10 microns were produced through melt spinning [20]. Some fibers were also subjected to a subsequent hot draw process, and then combined to form fiber tows of approximately 1500 filaments. These fibers were then stabilized within 30-180 minutes in a continuous process by passing fiber tows through multiple vessels containing fuming sulfuric acid and sulfuric acid with concentrations greater than $96 \%$, at temperatures ranging from $50-140{ }^{\circ} \mathrm{C}$, and applied tensions greater than $1 \mathrm{MPa}$. Fibers were then rinsed with deionized water and dried under vacuum overnight [21]. Stabilized fibers were then converted into carbon fibers by heating at temperatures ranging from 900 to $2400{ }^{\circ} \mathrm{C}$ in a nitrogen purged atmosphere. Three separate types of ovens were used, depending on the carbonization temperature required, including a Lindberg type 54459 (maximum temperature $1500{ }^{\circ} \mathrm{C}$ ), Micropyretics Heating International (MHI) model H18-40HT (maximum temperature $1760{ }^{\circ} \mathrm{C}$ ), and a Kanto Yakin Kogyo (KYK) model E-GRT-GRA-N2-0060-0060 (maximum temperature $2500{ }^{\circ} \mathrm{C}$ ). 
The properties of the 144 carbon fiber filaments studied in this work are shown graphically in Figure 4. Fibers with diameters ranging from $\sim 6-19$ microns, tensile moduli ranging from $\sim 35$ - $390 \mathrm{GPa}(\sim 60$ - $540 \mathrm{GPa}$ corrected for porosity), and tensile strengths ranging from $0.37-2.1$ $\mathrm{GPa}(0.59-2.9 \mathrm{GPa}$ corrected for porosity) were produced for this study at temperatures ranging from 900 to $2400{ }^{\circ} \mathrm{C}$.
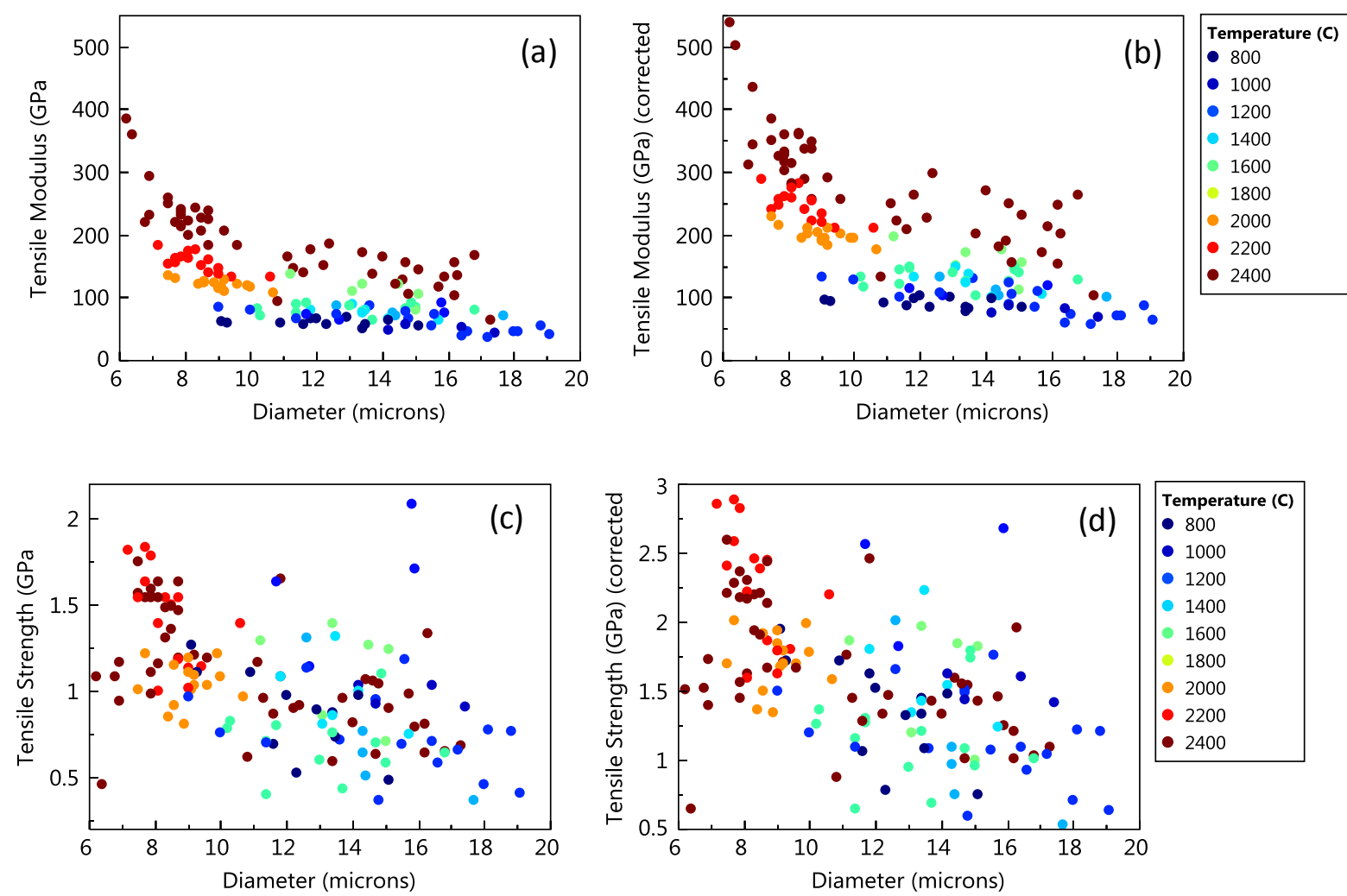

Figure 4. Plots of tensile moduli, strengths and diameter of the 144 carbon fiber filaments measured in this study, color-coded by ultimate carbonization temperature. Note that values are given (a), (c) asmeasured, and (b), (d) corrected for porosity.

\subsection{Single Filament Tensile Measurements}

Tensile modulus, strength, and elongation at break for individual, tab-mounted, carbon fiber filaments were measured using a dual column Instron frame (Model 5965) equipped with a 100 $\mathrm{N}$ load cell and controlled by Bluehill 2 software. The specific tensile testing methodology followed ASTM C1557 (Standard Test Method for Tensile Strength and Young's Modulus of Fibers). The filaments to be tested were selected at random from larger fiber tows following carbonization. This method specifies that single filaments are first mounted on paper or 
cardboard tabs with a center hole or longitudinal slot of fixed gage length; $2.54 \mathrm{~cm}$ was the exclusive gage length used in this work. As shown in Figure 5, the filament is laid down the center of the tab and held with tape at both ends. A small droplet of quick-curing epoxy is placed on both ends of the $2.54 \mathrm{~cm}$ gage length, which permanently bonds the filament to the tab. The diameter of all carbon fiber samples was measured via a laser diffraction technique [24-26]. For each sample, 20 single filaments were typically mounted; at least 15 of these were characterized for diameter and subjected to tensile testing.

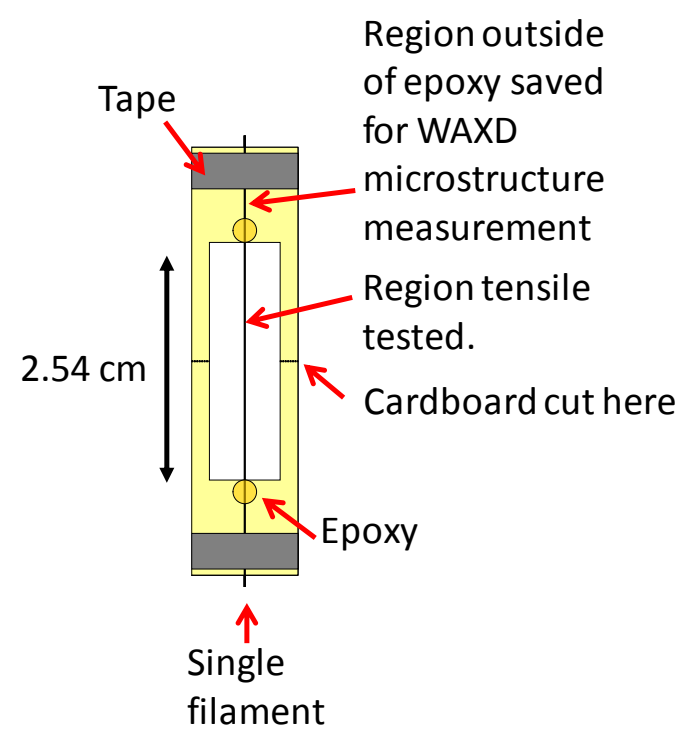

Figure 5. Schematic diagram of the cardboard tab used for mounting single carbon fiber filaments for tensile testing with the Instron.

The tab-mounted fibers were loaded into serrated-faced pneumatic grips of the Instron so that the carbon filament was well aligned with the vertical force direction of the frame. The two sides of the mounting tab were carefully cut, the load cell was zeroed, and a preload of 0.5 grams force was applied to the sample. Tensile testing was initiated at a constant crosshead speed of 0.5 $\mathrm{mm} / \mathrm{min}$ and continued until a fiber break was detected. The measured force was converted to stress using the fiber diameter. Sample strain was determined directly from the displacement of the crosshead. The system compliance of the load frame had been calculated for commercial Toray T300 carbon fibers at various gage lengths using the procedure outlined in ASTM C1557, and found to be very small compared to the variability in measured values, so this correction was 
not applied. The resulting stress-strain curves for the experimental fibers were typically highly linear, and the tensile modulus was calculated via the tangent method and reported in GPa units.

To be consistent with prior literature data, the tensile modulus was also corrected for porosity, according to

$$
E_{\text {Corrected }}=\frac{\rho_{\text {Crystalline }}}{\rho_{\text {Flotation }}} E_{\text {Measured }}
$$

where $\rho_{\text {Crystalline }}$ is the graphitic crystalline density and $\rho_{\text {Flotation }}$ is the bulk fiber density as measured by flotation using the density-gradient method. The crystalline density, which is the density of the graphitic material, not including porosity was calculated from WAXD measurements using both the (002) and (100) planar spacings, through the equation

$$
\rho_{\text {Crystaline }}=\frac{n M}{N_{a}\left(\frac{4}{3} \sqrt{3} d^{2}(100) d(002)\right)}
$$

where $n=4$ is the number of carbon atoms in the graphite hexagonal unit cell, $M=12$ is the molar mass of carbon, $N_{a}$ is Avogadro's number, and $d$ is the planar spacing of the (100) and (002) planes as obtained from WAXD measurements. 


\subsection{Wide-angle x-ray diffraction}

The microstructure of individual carbon fiber filaments was investigated using wide-angle $\mathrm{x}$-ray diffraction (WAXD) measurements conducted at the Advanced Photon Source (APS), DNDCAT, 5-ID-D beamline. Previously tensile-tested single filaments were selected and mounted on a custom aluminum stick. These were measured in vacuum in transmission-normal geometry using the standard APS Undulator A x-ray source at $17 \mathrm{keV}(\lambda=0.7293 \AA)$. Error! Reference source not found. shows the portion of fiber saved on each mounting card for WAXD measurements in relation to the $2.54 \mathrm{~cm}$ gauge length that was tensile tested with the Instron. Figure 6 shows images of single fiber filaments still attached to their cards mounted on an aluminum stick in addition to the chamber used to hold the fibers under vacuum during measurements, and the x-ray flight path. A stick can accommodate up to 30 filaments; however, alternating slots were generally left empty to accommodate the cardboard remaining from the original tensile test card. Two-dimensional diffraction patterns were collected on a Rayonix MarCCD 2k x 2k camera with acquisition times set at 3-5 minutes, depending on the fiber diameter and microstructure quality. Angular calibration of the detector was conducted using a lanthanum hexaboride powder standard. The sample-to-detector distance was set such that the entire graphite (004) peak was captured, typically $\sim 18 \mathrm{~cm}$ or less. Diffraction patterns were analyzed using the Dow-developed SCATTER data visualization and analysis package on the PV-WAVE platform [19], and JADE diffraction software [22]. Graphitic microstructure orientation was evaluated from the azimuthal intensity distribution of the (002) diffraction ring, as described herein. 

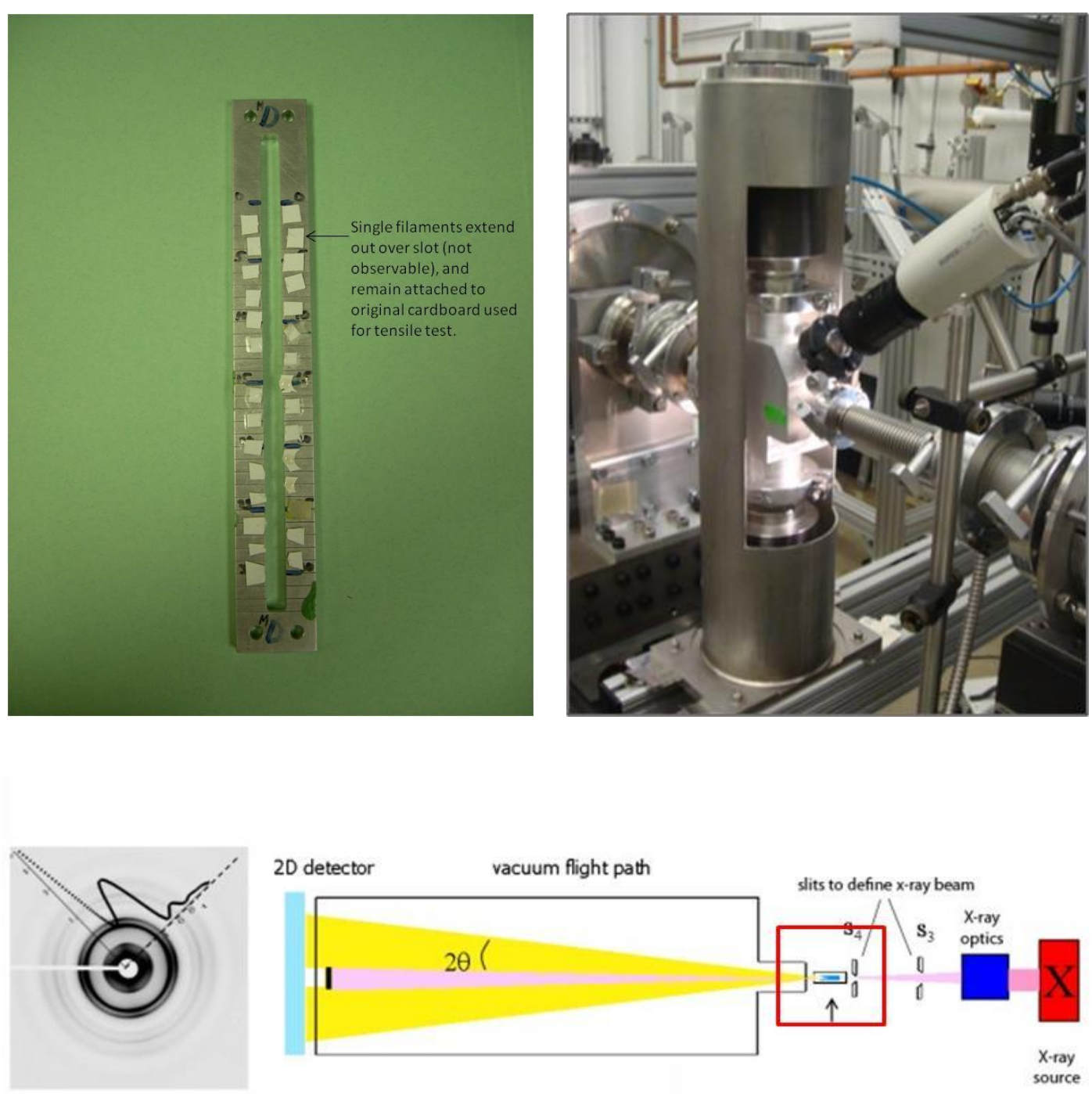

Figure 6. (top left) Image of carbon fiber filaments mounted on an aluminum stick. (top right) Custom chamber used to hold aluminum stick under vacuum during WAXD measurements. (bottom) X-ray flight path from source to detector. The red box indicates location of the vacuum sample chamber.

\subsection{Measurement of orientation}

The average orientation of graphitic layer plane normals relative to the fiber axis was evaluated indirectly from the azimuthal intensity distribution of the graphite (002) diffraction ring. Specifically, the intensity distribution of scattering from the (002) diffraction ring was evaluated relative to the meridional direction, yielding a $\left\langle\cos ^{2} \theta\right\rangle$ orientation parameter. The $\left.<\cos ^{2} \varphi\right\rangle$ orientation parameter was then calculated using the relationship

$$
\left\langle\cos ^{2} \theta\right\rangle=1-2\left\langle\cos ^{2} \varphi\right\rangle
$$


Due to the relatively weak scattering power of individual carbon fiber filaments, evaluation of orientation parameters required special attention to properly define the scattering intensity backgrounds and integration widths as described herein.

The first step in evaluating the orientation from a two-dimensional WAXD pattern required that the appropriate integration window (in degrees 2-theta) be chosen in order to capture the total anisotropy of the fiber microstructure. Specifically, the integration window was chosen so as to encompass the entire width of the (002) diffraction ring. Figure 7 shows the integration window (blue rectangle), which was determined separately for each fiber, as both the (002) diffraction peak position and width are unique to each fiber.
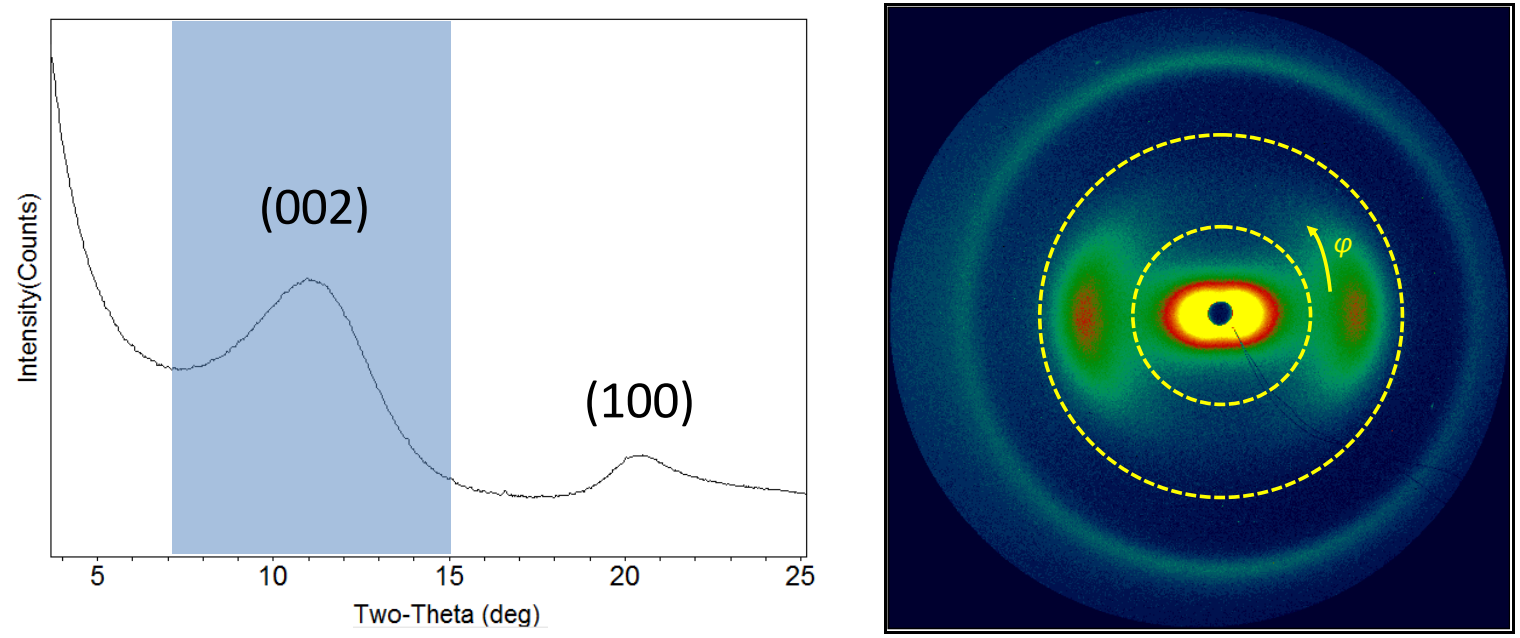

Figure 7. (Left) Scattering intensity vs. degrees two-theta obtained from integration of the WAXD pattern shown on the right. The blue window indicates the integration width used in evaluation of the microstructural orientation. (right) Two-dimensional WAXD pattern from a carbon fiber oriented with fiber axis along the vertical direction of the page. Graphitic layer normals are preferentially aligned in the equatorial direction. The yellow dotted circles correspond to the width of the blue box.

The intensity integrated across the specified 2-theta range of the (002) diffraction peak was then extracted and plotted as a function of azimuthal angle $\left(\varphi=0\right.$ to $\left.360^{\circ}\right)$, as shown in Figure 8 . The two peaks correspond to the two (002) diffraction peak maxima observed along the equatorial direction. Orientation parameters were numerically evaluated using the Dow-developed SCATTER data visualization and analysis package on the PV-WAVE platform over four 
regions: $90^{\circ}-0^{\circ}, 90^{\circ}-180^{\circ}, 270^{\circ}-180^{\circ}$, and $270^{\circ}-360^{\circ}$, and then averaged to yield a single value. Background position (shown in red) was determined from the average background over the predefined two-theta integration window range from an intensity slice taken along the meridional direction (along the fiber axis). The right plot in Figure 8 shows the presence of (002) peak intensity above the background, which indicates that highly unoriented graphene layers (perpendicular to fiber axis) are present. Thus the average background subtracted from the azimuthal intensity profile is slightly offset below the azimuthal intensity profile minimum by an amount equal to the (002) peak height above the background as shown in Figure 8.

In high modulus fibers, predominately all microstructure is found to be highly aligned with the fiber axis and thus no measurable (002) diffraction peak intensity is observed along the meridional direction, as shown in Figure 9. As a result, zero background offset is required for evaluation of the orientation parameter from the azimuthal intensity profile. However, it was found that the noise level of azimuthal intensity profiles was sufficiently high to artificially decrease the calculated orientation parameter from its actual value. Specifically, a background that cut through the middle of the noisy profile, still yielded significant intensity along the meridional direction, and resulted in an orientation parameter lower than the actual value. Thus, it was necessary to manually smooth the intensity profile between each azimuthal (002) peak, as shown in Figure 9. In this way, exactly zero intensity was achieved along the meridional direction, and the proper orientation parameter could be determined. This manual curve smoothing procedure was only required for the high-modulus fibers, where zero (002) diffraction intensity was observed along the meridional direction.
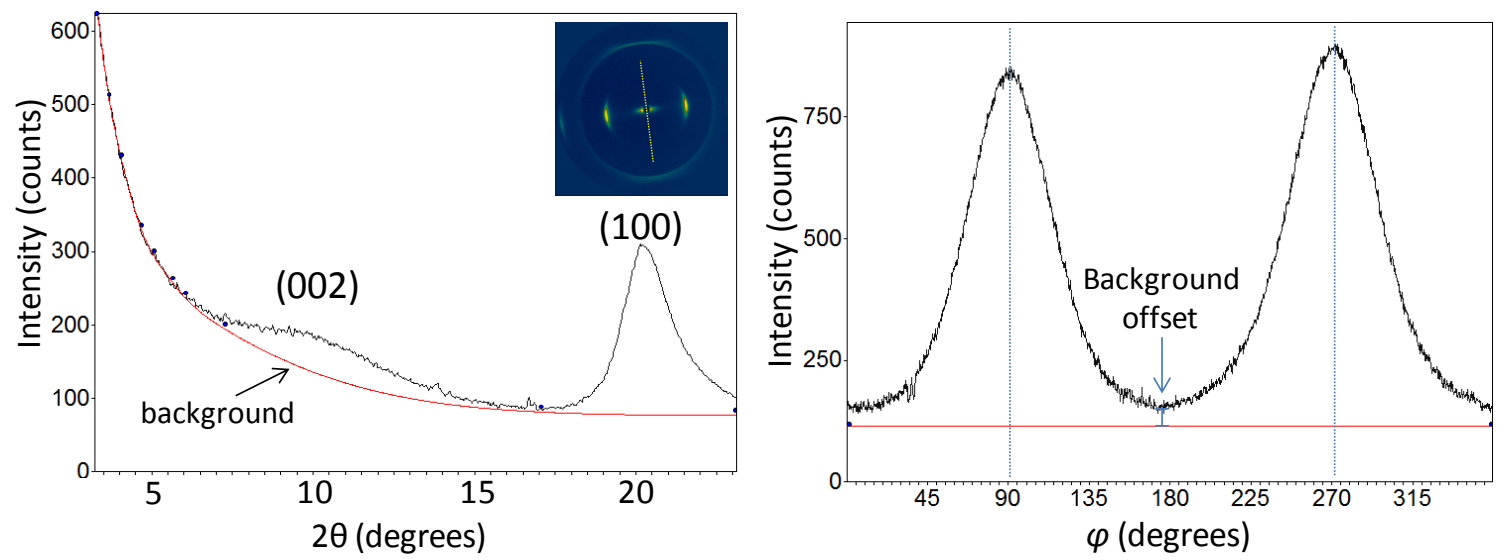
Figure 8. (left) Meridional intensity vs. two-theta (obtained along yellow line in inset WAXD pattern) scattering angle shows the presence of a (002) peak above background, indicating that highly unoriented microstructure is present in this particular fiber. The average background over the given two-theta range was used to establish the background for the azimuthal intensity profile shown on the right. (right) Azimuthal intensity variation of the (002) diffraction ring extracted from the WAXD pattern shown in Figure 7.
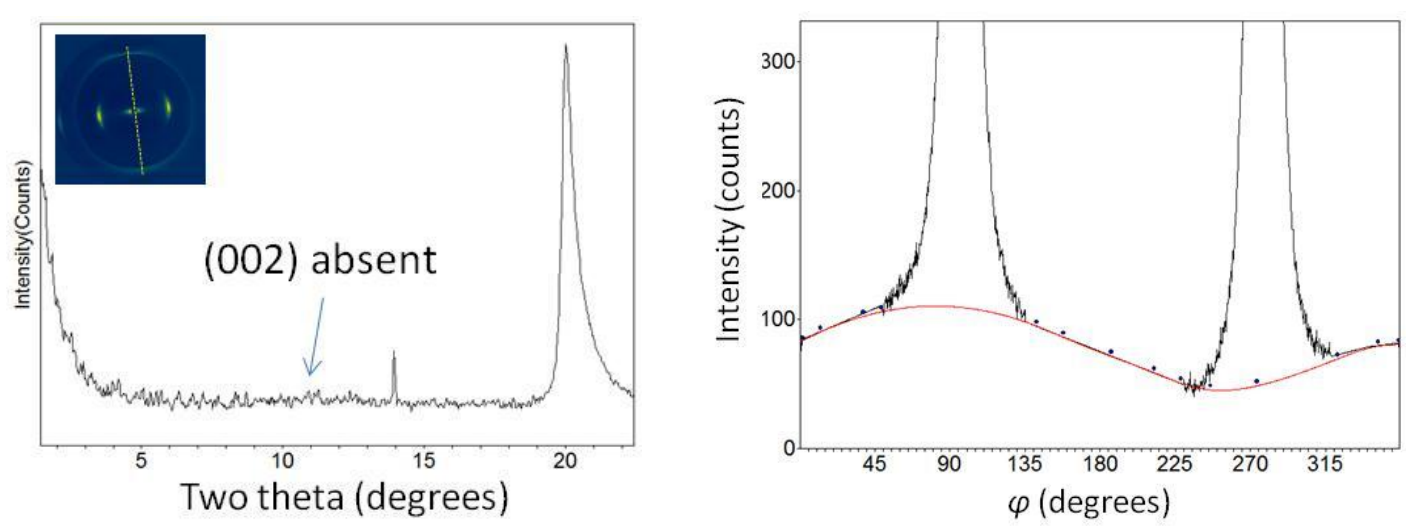

Figure 9. (Left) Meridional slice (along the dotted yellow line in inset WAXD pattern) of scattered intensity as a function of degrees two-theta for a high-modulus fiber does not show a (002) peak. (right) A magnified view of the azimuthal intensity profile showing a zero-offset red background. Note that the varying background is due to fluorescence occurring in the vacuum chamber upstream from the sample.

\subsection{Focused ion beam cross-section preparation for TEM}

The filaments were first mounted in epoxy with the fiber axis vertically oriented in the mount to expose transverse cross sections on the surface of the polished mount. Thin sections were cut from the polished surface of the mount with an FEI DB-235 dual beam focused ion beam scanning electron microscope (FIB-SEM) serial number 002301. The software used was FEI's TEM Wizard Deluxe Runscript TEM 3.03. Input parameters were: 20 micron foil length, 0.8 micron thick Pt-Ga-C ion deposited protective layer, 0.4 micron foil thickness, depth of 6 microns, and a mill factor of 2 . This process used the $30 \mathrm{kEV} \mathrm{Ga}$ ion beam with rough cuts at $5000 \mathrm{pA}$, and progressively finer cuts made at $3000 \mathrm{pA}, 1000 \mathrm{pA}$ and finishing the automated routine at $300 \mathrm{pA}$. Final thinning was done with the $\mathrm{Ga}$ beam at $10 \mathrm{keV}$ and approximately 50 pA for 30 to 60 seconds on each side of the foil. 


\section{Results and Discussion}

\subsection{Measurement system variability}

This study utilized three key measurement systems to evaluate the structure-property relationship for polyethylene-derived carbon fiber, including laser diffractometry to measure the fiber diameter, an Instron to measure the tensile force and strain, and WAXD to measure the microstructure orientation. The accuracy and precision of each of these measurement systems was evaluated, as described below.

A de-sized Toray T300 fiber sample was used to evaluate the laser diffractometry and Instron measurement systems [23]. As both of these measurement systems are used in the measurement of the tensile modulus, their composite error was determined through multiple single-filament tensile tests of a T300 bundle. Since a tensile test by nature is destructive to the sample, the same exact filament could not be re-tested. Instead, multiple 1-meter lengths of individual fiber filaments were extracted from the bundle, and then each was divided into 20 pieces for tensile measurement, using a standard $2.54 \mathrm{~cm}$ gauge length. Figure 10 shows the tensile moduli of Toray T300 single filaments measured in this study. The mean value of $225 \mathrm{GPa}$ agrees well with the tensile modulus for T-300 carbon fiber published by Toray (230 GPa) [27]. The relative standard deviation associated with this measurement technique was determined to be $\sim 10 \%$.

The precision of the corresponding WAXD technique for measurement of the orientation parameters was evaluated using a single filament of Dow PE-derived carbon fiber. The uncertainty introduced by variation in microstructure and data-processing were evaluated together by measuring the orientation parameter at multiple sites along the length of a single carbon fiber filament. The measured orientation parameters, each separated by $\sim 1 \mathrm{~cm}$ along the axis of a single PE-derived carbon fiber filament, and their corresponding distribution are shown in Figure 11. The observed random distribution with position suggests that the observed variation in orientation parameter is primarily a result of the measurement system. The relative standard deviation associated with this technique was determined to be $\sim 3.5 \%$. 

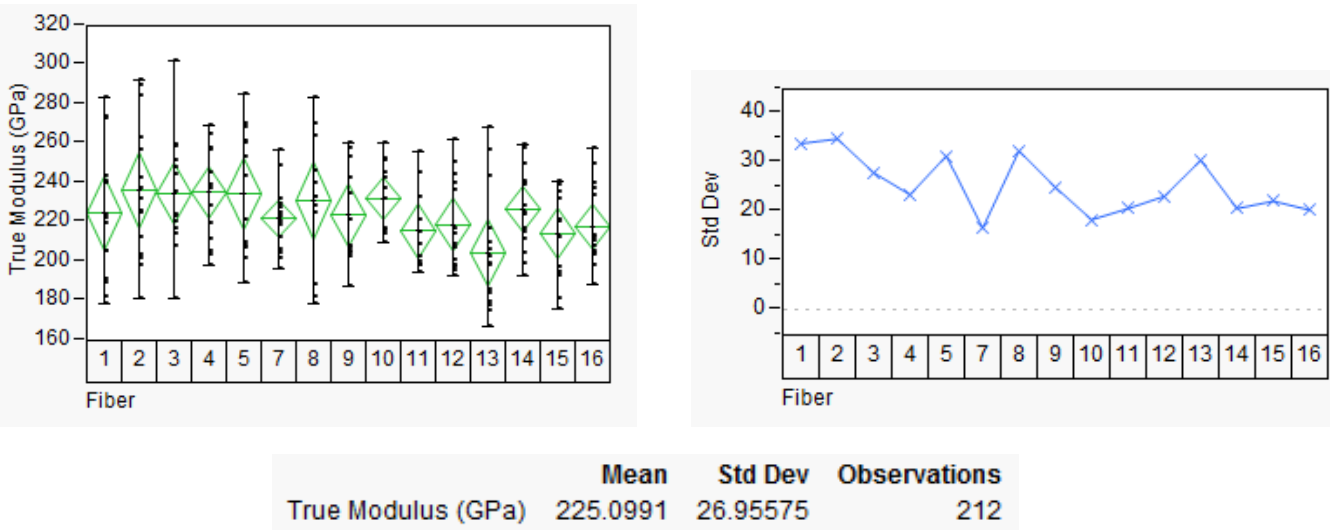

Figure 10. (Left) Tensile modulus and (right) standard deviation of de-sized Toray T300 filaments plotted for separate 1-meter pieces.
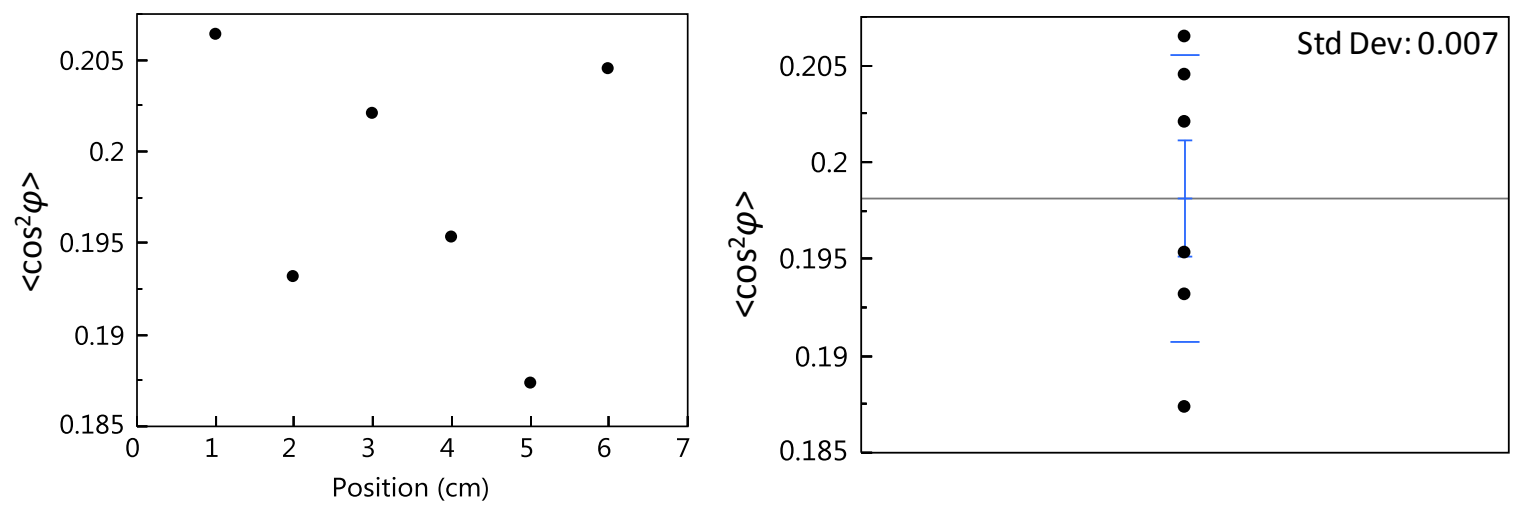

Figure 11. (Left) Microstructure orientation parameters measured as a function of position along a single PE-derived carbon fiber. (right) Distribution of measured microstructure orientation parameters.

\subsection{Structure-property relationship}

The relationship between graphitic layer orientation and the tensile modulus for polyethylenederived carbon fiber was determined from measurement of carbon fiber yarns consisting of 1573 filaments. As these yarns were carbonized over a wide range of temperatures, from 900 to 2400 ${ }^{\circ} \mathrm{C}$, both the final diameter and tensile moduli spanned a wide range. Figure 4 shows a plot of the diameter and corresponding tensile modulus for all carbon fibers examined in this study. Fiber diameters ranged from $\sim 6-19$ microns, tensile moduli ranged from $\sim 35-390 \mathrm{GPa}(\sim 60-$ $540 \mathrm{GPa}$ corrected for porosity), and tensile strengths ranged from $0.37-2.1 \mathrm{GPa}(0.59-2.9$ GPa corrected for porosity). Fiber density as measured by the density-gradient method varied from $\sim 1.3-1.6 \mathrm{~g} / \mathrm{cm}^{3}$; for comparison, typical PAN-derived carbon fibers exhibit densities greater than $1.7 \mathrm{~g} / \mathrm{cm} 3[1]$. 
Both the modulus and the strength plotted in Figure 4 increase with decreasing fiber diameter. While the strength, which is dependent on the concentration of defects, is expected to increase with decreasing diameter, the modulus, which is a material parameter, should be invariant with fiber diameter. Careful inspection of the modulus data; however, show that the smallest diameters and virtually all fiber with modulus above $100 \mathrm{GPa}$ were produced at the highest carbonization temperatures $\left(1800-2400^{\circ} \mathrm{C}\right)$. As summarized by Morgan [1], prior work on carbon fibers derived from both PAN and cellulose had demonstrated that hot stretching of carbon fibers at temperatures above $2000{ }^{\circ} \mathrm{C}$ led to significant increases in modulus and concomitant reductions in fiber diameter. Figure 4 shows that carbon fibers derived from polyethylene appear to respond in a similar manner.

Figure 12 shows the measured data for all of the carbon fibers, plotted as the fiber compliance $(1 / E)$ as a function of the orientation parameter, $\left\langle\cos ^{2} \varphi\right\rangle$. These data were then fit, to the uniform stress model in Eq. (1).

The model fit is relatively good, with an $\mathrm{R}$ square value of 0.74 . The $\mathrm{y}$-intercept of this fit, at $\left\langle\cos ^{2} \varphi>=0\right.$, corresponding to a perfectly oriented microstructure along the fiber axis, yields an in-plane tensile modulus of $e=960+/-300 \mathrm{GPa}$. This value is close to the theoretical in-plane tensile modulus of $1060 \mathrm{GPa}$ for bulk graphite [15], and indicates that if a perfectly-orientated microstructure could be achieved, the resulting fiber tensile modulus would be $960 \mathrm{GPa}$. The shear modulus for shear between adjacent graphene layers, obtained from the slope of the fit line, gives a value of $g=24+/-1 \mathrm{GPa}$, which is significantly larger than the shear modulus for bulk graphite (i.e., $g=4-5 \mathrm{GPa}$ ).

A shear modulus greater than that for bulk graphite has been observed previously in carbon fibers produced from PAN, pitch and cellulose [7, 14]. As discussed by both Northolt et al. and Sauder et al., values above $g_{\text {graphite }}$ can be attributed to the presence of covalent C-C $\mathrm{sp}^{3}$ crosslinks that connect adjacent graphitic layers within the carbon fiber. Disorder in the graphitic structure alone does not increase shear modulus. In fact, the shear modulus of defectfree graphite is expected to decrease when disorder or defects are introduced into the lattice [15]. 
For comparison, diamond, a purely $\mathrm{sp}^{3}$-bonded carbon structure, exhibits a very high shear modulus of $577 \mathrm{GPa}[15]$.

Formation of covalent crosslinks between adjacent graphitic layers is largely governed by the chemical and structural nature of the fiber precursor and the stabilization processes used. As a result, the shear modulus, and thus the relationship between microstructure orientation and the tensile modulus are unique to a particular type of carbon fiber. For comparison, orientationmodulus data for PAN- and pitch-derived carbon fibers was compiled from numerous sources in the published literature [1,7-11]. These data are shown in the plot of Figure 12, along with the orientation-modulus data for PE-derived carbon fiber measured in this work. From this plot, it is evident that the relationship between orientation and modulus is dependent on the precursor type. Fibers produced from PAN exhibit the highest shear moduli, $g=46+/-10 \mathrm{GPa}$ while both pitchand PE-derived carbon fiber exhibit significantly lower shear moduli, $\mathrm{g}=35+/-1 \mathrm{GPa}$ and $\mathrm{g}=$ $24+/-1 \mathrm{GPa}$, respectively. This trend $\left(g_{P A N}>g_{\text {pitch }}\right)$ is consistent with that observed previously by both Northolt et al and Sauder et al [7, 18]. It should be noted that the Northolt model has been shown to underestimate shear modulus, however this does not change the observed trend and conclusions drawn from this work. Carbon fiber derived from mesophase pitch is expected to exhibit relatively few covalent crosslinks, because of the extended cyclic, polyaromatic structure present in this precursor. In this way, PE-derived carbon fiber is much more similar to pitch than it is to PAN, despite the significant structural differences at the precursor level. In contrast to pitch, PAN precursor does not contain aromatic rings. This combined with the specific oxidation stabilization processes used leads to high levels of crosslinks are formed between adjacent chains as the precursor is converted to the polyaromatic structure of carbon fiber.

These fundamental differences have important consequences on the resulting mechanical properties. For example, for an equal degree of microstructure orientation, PAN-derived carbon fiber will exhibit higher tensile modulus than pitch-derived carbon fiber. However, it is also much more difficult (higher temperatures and tensions are required) to orient the microstructure in a PAN-derived carbon fiber in comparison to pitch- or PE-derived fibers because of the higher levels of covalent crosslinks in the PAN-derived fiber. As a result, much higher carbonization 
temperatures and tensions are required to obtain high modulus PAN-based carbon fiber [10]. Second, a strong correlation between the shear modulus and compressive strength has been observed previously [7]. In fact, the compressive strength of PAN-derived carbon fibers have been found to be significantly greater than those of pitch-derived fibers [24].

As shown in Figure 12, the PE-derived carbon fibers measured in this study exhibit a shear modulus even lower than that of pitch-derived carbon fiber. This indicates that compared to pitch-derived fiber, the PE-derived fiber contains even fewer covalent $\mathrm{C}-\mathrm{C} \mathrm{sp}^{3}$ crosslinks. Thus, it is anticipated that these fibers might exhibit compressive strengths lower than for pitch-derived fibers. However, a structure with fewer covalent crosslinks should graphitize more easily, yielding a higher level of microstructural orientation at a given temperature. Indeed, it has been observed that the graphitization behavior of PE-derived carbon fiber is accelerated in comparison to that of PAN and pitch fibers, but this will be discussed in a future publication.

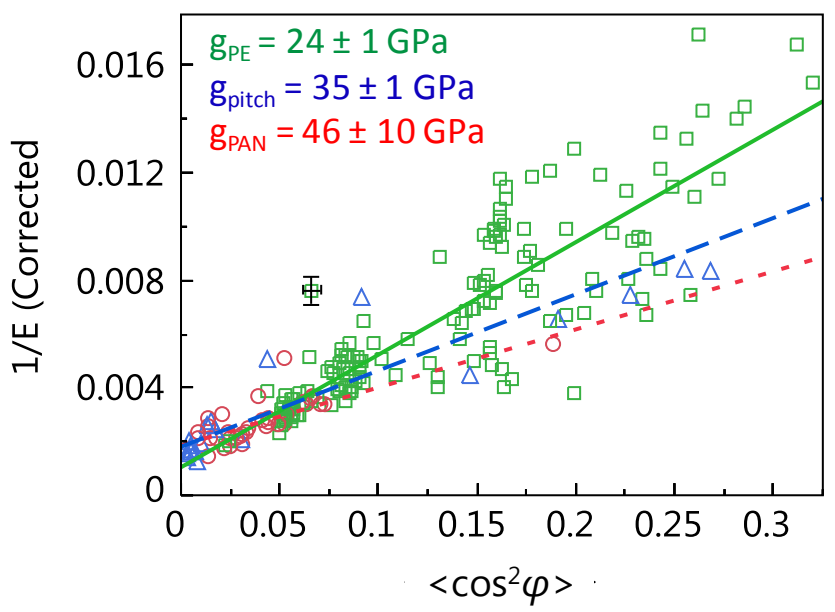

Figure 12. Plots of compliance (1/E) vs. orientation parameter for PE- (green squares), PAN- (red circles), and pitch- (blue triangles) derived carbon fiber. Calculated shear moduli for each type are listed in the same order. Tensile moduli for the PE-derived carbon fiber are corrected for porosity. Error bars were estimated from replicated measurements of the same fiber.

\subsection{Additional sources of error}

As shown in Figure 12, significant scatter is observed in the data, which cannot be fully accounted for by the $\sim 10 \%$ relative error in (uncorrected) modulus measurement, and $\sim 4 \%$ 
relative error in orientation parameter measurement by WAXD. Still, there are a number of other factors that could significantly affect the accuracy of a tensile modulus measurement for a particular individual filament. Specifically, it has been often observed that pores are present at some interior locations within the fibers, as shown in Figure 13. The size and density of these pores vary along the length of a filament, which results in a change of the effective crosssectional area of the filament. Typical pore sizes range from nanometers to $\sim 1$ micron in diameter. As the tensile modulus is calculated using the cross-sectional area determined from fiber diameter, the presence of pores within the tested gauge length could result in significant modulus error. Density measurements were utilized in this study to correct for porosity; however, density measurements were an average of the entire bundle, and thus would not capture variation in porosity among or within fibers. This error should not have an impact on the orientation parameter measurements, however.

A second possible source of error could result from a non-uniform graphitic structure across the fiber diameter. Cross-sectional scanning electron micrographs of fracture surfaces of fibers after tensile failure (Figure 13) show surfaces that are jagged and exhibit patterns akin to growth rings across a tree trunk. Further, this tree-ring like pattern is not evident near the central core of the fiber, suggesting a core-sheath effect where the structure and properties in the outer layers may differ significantly from the central axis of the fiber. 

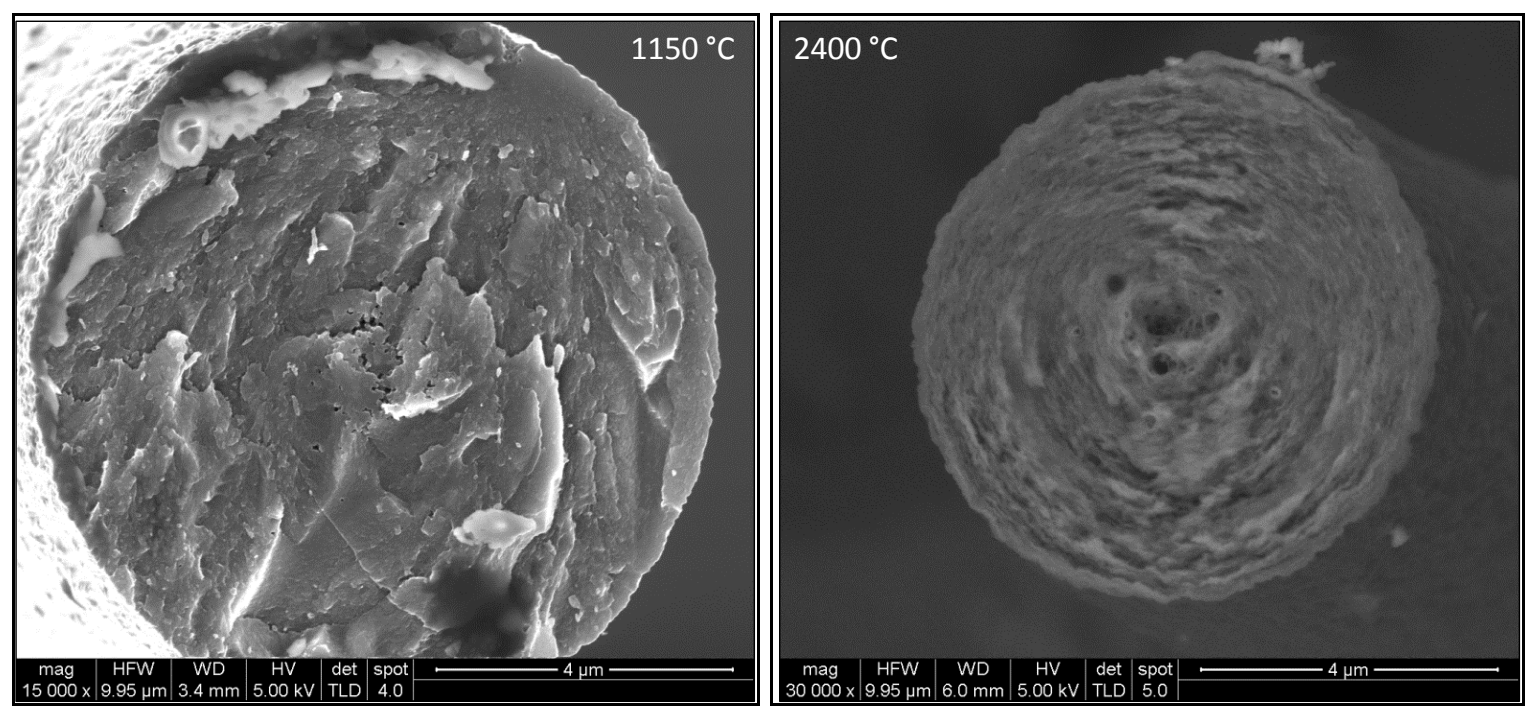

Figure 13. Secondary electron microscopy images of the fracture surfaces of carbon fibers pulled in tension. The temperature indicates the final carbonization temperature for each fiber. Pores in both fibers, and a tree-ring like morphology in the high-temperature fiber are clearly observed.

Indeed, cross-sectional transmission electron micrographs and selected area electron diffraction measurements, as shown in Figure 14, reveal that the inner $\sim 2$ micron core of a fiber carbonized to $2400{ }^{\circ} \mathrm{C}$ exhibits lower microstructural orientation than the outer sections of the fiber. The result of this heterogeneity is that the poorly-oriented central core may not contribute to the measured fiber tensile modulus, but does in fact contribute to the orientation parameter determination. Thus, the measured orientation parameter will be lower than the actual orientation of the outer microstructure that governs the tensile modulus. This yields a higher calculated shear modulus, thus the true shear modulus for PE-derived carbon fiber is likely to be less than the $24 \mathrm{GPa}$ reported herein. Variability in the volume fraction of less-oriented core microstructure may help explain the observed data scatter. 

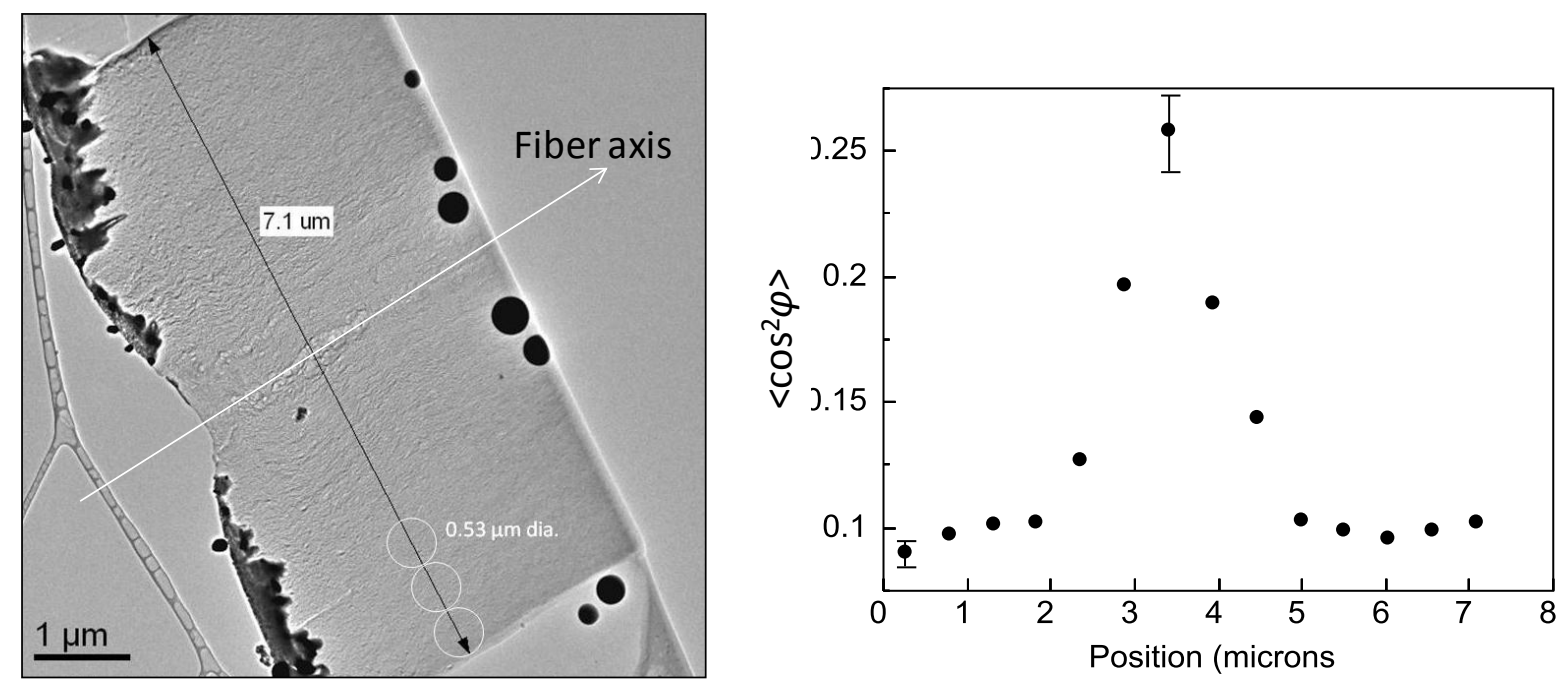

Figure 14. (Left) Transmission electron micrograph of a cross-section through the center of a carbon filament. White circles show selected-area aperture size, and first three locations across the fiber diameter from which diffraction patterns were obtained. (right) Orientation parameter as a function of radial position across the fiber obtained by electron diffraction shows lower microstructure orientation near the fiber core.

This core-sheath structure, especially for fibers carbonized at higher temperatures, may originate from the hot stretching as discussed above. For the short sections of experimental fiber used in this work, samples were typically tied into commercial carbon fiber leaders with knots, which resulted in non-uniform tension across the experimental fiber tow. The variation in tension amongst the filaments presumably contributed additional variability to the moduli and diameters since the filaments under the highest tension experienced more elongation, resulting in higher moduli and smaller diameters. This effect is not seen at lower carbonization temperatures where stretching is absent.

\section{Conclusions}

In this work, the uniform stress $[7,11]$ structure-property model that relates the tensile modulus to the elastic properties and angular distribution of the constituent graphitic layers was applied to carbon fiber derived from polyethylene precursors. Density measurements to correct for porosity were found to be critical to accurately describe the true relationship between the tensile modulus and microstructure orientation, and to enable comparison to published modulus-orientation data 
for PAN- and pitch-derived carbon fibers. The predictive capability of this model enables calculation of the required microstructure orientation level to achieve a given tensile modulus, or the expected tensile modulus for a given level of microstructure orientation. New synchrotron WAXD methodologies developed enabled measurement of the microstructure orientation of the exact single filaments that had been previously tensile-tested. Analysis of the data using this uniform-stress structure-property model revealed fundamental differences in the nature of the microstructure present in carbon fiber produced from polyethylene, as compared to carbon fibers produced from polyacrylonitrile (PAN) or pitch precursors. Specifically, it was found that the shear modulus for shear between adjacent graphitic layers is lower for polyethylene-derived carbon fiber than for PAN- or pitch-derived carbon fibers, suggesting that covalent $\mathrm{C}-\mathrm{C} \mathrm{sp}^{3}$ crosslink density in PE-derived carbon fiber is reduced. However, this less crosslinked structure is anticipated to be easier to orient during carbonization and high-temperature graphitization processes, yielding a highly oriented structure, which is essential for achieving high tensile modulus. Future work will focus on in situ investigation of graphitic microstructure development during carbonization and high-temperature graphitization processes.

\section{Acknowledgements}

Portions of this work were performed at the DuPont-Northwestern-Dow Collaborative Access Team (DND-CAT) beamline line located at Sector 5 of the Advanced Photon Source (APS). DND-CAT is supported by E.I. DuPont de Nemours \& Co., The Dow Chemical Company and Northwestern University, as well as the U.S. Department of Energy under Contract No. DEAC02-06CH11357. The authors also acknowledge funding support from the 21 st Century Jobs

Trust Fund received through the Michigan Strategic Fund from the State of Michigan (Grant \# DOC-2868).

\section{References}

[1] Morgan P, Carbon fibers and their composites. Boca Raton FL: Taylor \& Francis, LLC. 2005.

[2] Horikiri SA, Iseki J, Minobe M. Process for production of carbon fiber. US patent 4070446, 1978. 
[3] Postema AR, De Groot H, Pennings AJ. Amorphous carbon fibres from linear low density polyethylene. Journal of Materials Science 1990; 25: 4216-4222.

[4] Penning JP, Lagcher R, Pennings AJ. The effect of diameter on the mechanical properties of amorphous carbon fibres from linear low density polyethylene. Polymer Bulletin 1991; 25: 405412.

[5] Zhang D, Sun Q. Structure and properties development during the conversion of polyethylene precursors to carbon fibers. Journal of Applied Polymer Science 1996; 62: 367-373.

[6] Zhao H, Min K, Aluru NR. Size and chirality dependent elastic properties of graphene nanoribbons under uniaxial tension. Nano Letters 2009; 9(8): 3012-3015.

[7] Northolt MG, Veldhuizen LH, Jansen H. Tensile deformation of carbon fiber and the relationship with the modulus for shear between the basal planes. Carbon 1991; 29(8): 12671279.

[8] Fischer L, Ruland W. The influence of graphitization on the mechanical properties of carbon fibers. Colloid and Polymer Science 1980; 258(8): 917-922.

[9] Loidl D, Peterlik H, Muller M, Riekel C, Paris O. Elastic moduli of nanocrystallites in carbon fibers measured by in-situ X-ray microbeam diffraction. Carbon 2003; 41(3): 563-570.

[10] Isaac DH, Ozbek S, Francis JG. Processing of carbon fibers: texture enhancement induced by hot stretching. Materials and Manufacturing Processes 1994; 9(2): 179-197.

[11] Ruland W. The relationship between preferred orientation and young's modulus of carbon fibers. ACS Polymer Preprints 1968; 9: 1368-1375.

[12] Huang Y, Young RJ. Effect of fiber microstructure upon the modulus of pan- and pitchbased carbon fibers. Carbon 1995; 33(2): 97-107.

[13] Bacon R, Schalamon WA. Physical properties of high modulus graphite fibers made from a Rayon precursor. Applied Polymer Symposia 1969; 9: 285-292.

[14] Sauder C, Lamon J. Prediction of elastic properties of carbon fibers and CVI matrices. Carbon 2005; 43(10): 2044-2053.

[15] Kelly BT. Physics of graphite. London: Applied Science Publishers.1981.

[16] Alexander LE. X-ray diffraction methods in polymer science. New York: WileyInterscience. 1969. 
[17] Wilchinsky Z. Recent developments in the measurement of orientation in polymers by $x$-ray diffraction. In: Mueller W, Fay M, editors. Advances in X-Ray Analysis, Springer; 1995, p. 231241.

[18] Sauder C, Lamon J, Pailler R. The tensile behavior of carbon fibers at high temperatures up to $2400{ }^{\circ} \mathrm{C}$. Carbon 2004; 42(4): 715-725.

[19] Barton BE, Patton JT, Hukkanen EJ, Behr MJ, Lin JC, Beyer S, Zhang Y, Brehm L, Haskins B, Bell B, Gerhart B, Leugers A, Bernius M. The chemical transformation of hydrocarbons to graphitic carbon using $\mathrm{SO}_{3}$ sources. Carbon 2015; 94: 465-471.

[20] Spalding MA, Wang W, Pavlicek CL, Horstman NJ. Small diameter polyolefin fibers. PCT /US patent application PCT/US2014/056538, 2015.

[21] Barton BE, Patton JT, Hukkanen EJ, Bernius MT. Two-step sulfonation process for the conversion of polymer fibers to carbon fibers. US patent application US 14/413,454, 2015. [22] Rudolf PR, Landes BG. Two-dimensional x-ray diffraction and scattering of microcrystalline and polymeric materials. Spectroscopy 1994; 9(6): 22-33.

[23] Dobb MG, Johnson DJ, Park CR. Compression behavior of carbon fibers. Journal of Materials Science1990; 25(2A): 829-834.

[24] Adedoyin AA, Li C. Towes MD. Characterization of single cotton fibers using a laser diffraction system. Textile Research Journal 2011; 81: 355-367.

[25] Li CT, Tietz JV. Improved accuracy of the laser diffraction technique for diameter measurement of small fibres. Journal of Materials Science 1990; 25: 4694-4698.

[26] Madronero A, Merino C. Some geometrical singularities in the characterization of vapor grown carbon fibers using laser diffraction technique. Materials Research Bulletin 1998; 33: 1503-1515.

[27] Torayca T300 Data Sheet, No. CFA-001, http://www.toraycfa.com/pdfs/T300DataSheet.pdf 